Check for updates

Cite this: RSC Adv., 2017, 7, 23427

\title{
Anisotropic atomic-structure related anomalous Hall resistance in few-layer black phosphorus
}

\author{
T. Makino, ${ }^{a}$ Y. Katagiri, ${ }^{a}$ C. Ohata, ${ }^{a}$ K. Nomura ${ }^{a}$ and J. Haruyama ${ }^{\star b}$
}

Mono- (or few) layer black phosphorus (BP) is highly expected to be a next-generation two-dimensional (2D) atom-thin semiconductor. Compared with other 2D atom-thin layers, BP has a significantly anisotropic atomic structure, which can provide unique electronic, optical, and magnetic properties. Herein, we report the observation of $B^{2 / 3}$ magnetic-field $(B)$ dependence of the Hall resistance with extremely small quantized levels in the low- $B$ regime of the few-layer BP, which are associated with the anisotropic electronic structure confirmed by largely anisotropic hole mobilities. One of the qualitative interpretations for the $B^{2 / 3}$ dependence is the possible presence of a uniaxial Dirac spectrum (arising from a closed band gap caused by uniaxial stretch along the buckling armchair direction, which is occasionally introduced by a process for cutting and wire-bonding samples, confirmed by Raman spectroscopy) and its hybridization with a Schrödinger spectrum existing along a zigzag direction. The observed phenomena imply significant potential due to strong anisotropy of atom-thin (few-layer) BP, which is highly expected to open doors to novel physics and its application for innovative 2D atom-thin devices.

Received 28th March 2017

Accepted 11th April 2017

DOI: $10.1039 / \mathrm{c} 7 \mathrm{ra03600k}$

rsc.li/rsc-advances structures, respectively, are different. Indeed, $|t|=1.220 \mathrm{eV}$ for the in-plane zigzag and $t^{\prime}=3.665 \mathrm{eV}$ for the buckling armchair, with the $m^{*}$ several times lighter in the armchair than in the zigzag direction, and a strong angular dependence of the conductivity has been reported in mono(few)-layer BP. ${ }^{6-8,12,27}$

This $t \neq t^{\prime}$ yields unique $B$ dependence of the Landau Levels (LLs) in graphene, ${ }^{\mathbf{1 4}}$ in which the energy levels around the band center $(E=0)$ (with Dirac spectrum, ${ }^{15}$ Fig. 1(c)) vary with $B$ as $E_{\mathrm{N}}(B) \sim \pm(\mathrm{NB})^{1 / 2}$, with a twofold valley degeneracy. ${ }^{16}$ This $B^{1 / 2}$ -

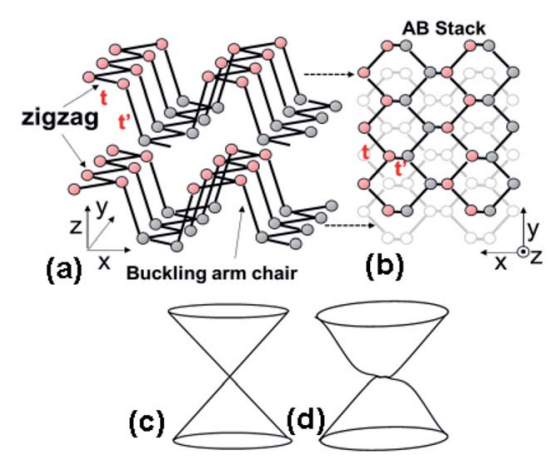

Fig. 1 Schematic of (a) in-plane anisotropic atomic structure of BP (i.e., zigzag within monolayer along the $y$-axis and buckling armchair formed over two layers along the $x$-axis) and (b) a puckered honeycomb lattice on a top view. $t$ and $t^{\prime}$ are hopping integrals between nearest neighbors, corresponding to $\mathrm{P}$-atoms within in-plane zigzag and buckling armchair over two layers, respectively. (c) and (d) examples of low energy spectrum for (c) $t^{\prime}=t$ and (d) $t^{\prime}=2 t$ in graphene.
${ }^{a}$ Faculty of Science and Engineering, Aoyama Gakuin University, 5-10-1 Fuchinobe, Sagamihara, Kanagawa 252-5258, Japan.E-mail: J-haru@ee.aoyama.ac.jp

${ }^{b}$ Institute for Solid State Physics, The University of Tokyo, 5-1-5 Kashiwanoha, Kashiwa, Chiba 277-8581, Japan tial energy band gap intermediating between the zero-gap graphene and the large band-gap of the transition-metal $\sim 4000 \mathrm{~cm}^{2} \mathrm{~V}^{-1} \mathrm{~s}^{-1}$ at low temperatures and Shubnikov-de Haas (SdH) oscillations have been demonstrated in few-layer BP with improved crystal quality resulting from the removal of impurities. ${ }^{3}$ Large edge-magnetism at room temperature has also been predicted and actually observed in oxygen-terminated $\mathrm{BP}$ nanoribbons and nanomeshes., ${ }^{4,5}$

Highly in contrast to graphene, mono(few)-layer BP has a unique anisotropic atomic structure (Fig. 1(a); i.e., a zigzag structure within a monolayer along the $y$-axis and buckling armchair formed over two layers along the $x$-axis), which results in a puckered honeycomb lattice on a top view (Fig. 1(b)). It yields anisotropic electronic structures (e.g., electron effective mass $m^{*}$ and $\mu$ ), which are very important for innovative electronic and optical device ${ }^{27}$ applications. As shown in Fig. 1(a) hopping integrals $t \neq t^{\prime}$ between the nearest neighbors because $t$ and $t^{\prime}$ for the in-plane zigzag and buckling armchair 
dependent spectrum is different from the well-known $B$ dependence of LLs; $E_{N}(B)=(N+1 / 2) e B / m^{*}$ for electrons in a quadratic band with $m^{*}$ (i.e., Schrödinger spectrum). On the other hand, the novel $B$ dependence of the LLs implying a power law, a $[(N+\gamma) B]^{2 / 3}$ behaviour with $\gamma=1 / 2$ arising from a hybridization of the Dirac and Schrödinger spectrum, has been theoretically reported recently for the specified graphene with $t \neq t^{\prime}$ (i.e., $t^{\prime}=2 t$; Fig. $1(\mathrm{~d})$ ) in the low $B$ region of the Hofstadter-Rammal spectrum. ${ }^{14,26}$ The value $\gamma=1 / 2$ results from the cancellation of a Berry phase.

Moreover, band gap closure resulting in the appearance of a Dirac cone ${ }^{28}$ has been recently reported in potassium-doped $\mathrm{BP}$, exhibiting strong anisotropy (i.e., a Dirac semimetal state with a linear band dispersion in the armchair direction and a quadratic one in the zigzag direction), ${ }^{\mathbf{9}, 10}$ as well as band gap modulation caused by strain. ${ }^{11-13}$ Therefore, it is crucial to experimentally clarify how the anisotropic atomic structures yield further unique physical characteristics, which contribute to the applications for innovative electronic, optical, and magnetic devices in mono(few)-layer BPs.

\section{Results and discussion}

\section{Sample characterization}

Fig. 2(a) and (b) show optical and atomic force microscope images of a BP with top and cross sectional views, respectively. The samples have been fabricated by mechanical exfoliation of bulk BP (Smart Element Co.) on a clean $\mathrm{SiO}_{2} / \mathrm{Si}$ substrate, of which the surface was thoroughly cleaned mechanically and chemically $\left(\mathrm{O}_{2}\right.$ plasma cleaning) before forming the BP in order to obtain high carrier mobility, following the Scotch tape method. Typical sample area and thickness are $\sim 400 \mu \mathrm{m}^{2}$ and $\sim 4 \mathrm{~nm}$, respectively.

After the mechanical exfoliation, the BP samples have been preserved in high-vacuum in order to avoid heavy oxidation.
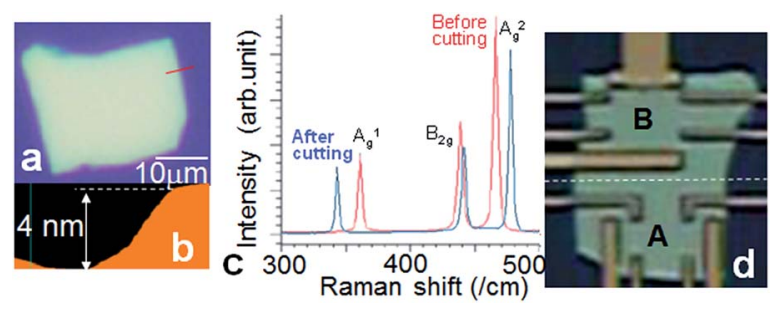

Fig. 2 (a) Optical and (b) atomic force microscope images of a BP flake on top and cross sectional views, respectively. Panel (b) has been measured along red line shown in (a) (i.e., at a right edge of the flake). (c) Typical Raman spectrum of the (a) sample corresponding to sample $A$ in (d). Red curve is the spectrum before cutting the substrate, and the blue curve is that after cutting the substrate into two individual substrates each with one Hall pattern, thinning, mounting on packages, and wire-bonding to the package by Au wires. (d) Optical microscope image of the sample with two Hall bar patterns with $90^{\circ}$ different directions ( $A$ and $B$ ) used for electrical measurements. White dotted line between two regions is just a guide for the eyes. Each sample direction is not intentionally aligned to crystal orientations $(x, y)$ shown in Fig. 1(a) and (b). As mentioned in (c), the substrate has been cut into two individual substrates for electrical measurements.
Fig. 2(d) shows an optical microscope image of the sample with two Hall bar patterns used for electrical measurements. Two Hall patterns have been fabricated on one BP substrate in different directions within $90^{\circ}$ (i.e., main bars of samples A and B) in order to clarify the anisotropic electronic transport, although each sample direction is not intentionally aligned to crystal orientations $(x, y)$ shown in Fig. 1(a) and (b). After this initial formation, the $\mathrm{Si} / \mathrm{SiO}_{2}$ substrate has been mechanically cut by dicing into two individual substrates each with one Hall pattern. Then, the individual $\mathrm{Si} / \mathrm{SiO}_{2}$ substrates have been thinned and mounted on packages for electrical measurements, and electrode pads have been bonded to the package by Au wires.

The red curve in Fig. 2(c) shows the Raman spectrum of the sample shown in Fig. 2(a) (sample A in (d)) before cutting the substrate, which agrees with that in few-layer BP. In contrast, the blue curve demonstrates the Raman spectrum after cutting this substrate into two individual substrates each with one Hall pattern by hand thinning, mounting on packages, and bonding to the package by Au wires (sample A in (d)). It implies a large shift of $\mathrm{A}_{\mathrm{g}}^{1}$ and $\mathrm{A}_{\mathrm{g}}^{2}$ peaks in opposite directions (i.e., 360 to $340 \mathrm{~cm}^{-1}(\sim-1 / 18)$ for $A_{\mathrm{g}}^{1}$ and 465 to $480 \mathrm{~cm}^{-1}(\sim 1 / 31)$ for $\left.\mathrm{A}_{\mathrm{g}}^{2}\right)$. Ref. 24 gives the quantitative information for changes of Raman shift ratios caused by strain modulation (i.e., $\sim-1 / 12$, $\sim 1 / 22$, and $\sim 1 / 15$ for $A_{g}^{1}, B_{g}^{2}$, and $A_{g}^{2}$, respectively). Our results for $A_{g}^{1}$ and $A_{g}^{2}$ peaks are qualitatively consistent with those considering the opposite shift direction, although the shift values are smaller than those in ref. 24. On the other hand, the $\mathrm{B}_{\mathrm{g}}^{2}$ peak is almost unchanged in Fig. 2(c). This is also consistent with the extremely small shift of the $\mathrm{B}_{\mathrm{g}}^{2}$ peak against the applied strain because $\mathrm{B}_{\mathrm{g}}^{2}$ mode originates from only lattice vibration in parallel with the substrate. Therefore, Fig. 2(c) suggests the introduction of a stretch mode (uniaxial strain) into few-layer BP, which should result in a closed band gap and subsequent appearance of the observed anisotropic Hall resistance (i.e., $B^{2 / 3}$ dependence due to the closed band gap) as shown later.

\section{Electrical properties}

Fig. 3 shows examples of typical electrical properties of two samples. Fig. 3(a) shows the ohmic feature for a drain current $\left(I_{\mathrm{DS}}\right)$ as a function of drain voltage $\left(V_{\mathrm{DS}}\right)$ of sample $\mathrm{A}$, whereas sample $\mathrm{B}$ shows much lower $I_{\mathrm{DS}}$. Interestingly, we find that field-effect mobility for the hole $\left(\mu_{\mathrm{h}}\right)$, which is extracted from the line fit of the transfer characteristics (i.e., relationships for normalized conductance $(G)$ vs. back gate voltage $\left.\left(V_{\mathrm{bg}}\right)\right)$, has strong anisotropy as shown in Fig. 3(b) (sample A) and 3(c) (sample B). In sample A, $\mu_{\mathrm{h}}$ is estimated to be $\sim 320 \mathrm{~cm}^{2} \mathrm{~V}^{-1} \mathrm{~s}^{-1}$ at $T=300 \mathrm{~K}$ and $\sim 3300$ $\mathrm{cm}^{2} \mathrm{~V}^{-1} \mathrm{~s}^{-1}$ at $T=1.5 \mathrm{~K}$ (from dotted lines in Fig. 3(b)), whereas much smaller $\mu_{\mathrm{h}}$ of $\sim 120 \mathrm{~cm}^{2} \mathrm{~V}^{-1} \mathrm{~s}^{-1}$ at $T=300 \mathrm{~K}$ is estimated in sample B (Fig. 3(c)). This $\mu_{\mathrm{h}}$ of $\sim 3300 \mathrm{~cm}^{2} \mathrm{~V}^{-1} \mathrm{~s}^{-1}$ almost agrees with that of previous reports of $\mathrm{BP}$, with the best quality reported in ref. 3 , and it allows the possible observation of low- $B$ (quantum) Hall resistances (particularly $R_{x y}$ ) at low temperatures. The abovementioned surface cleaning of the $\mathrm{Si} / \mathrm{SiO}_{2}$ substrate and a thickness of $\sim 4 \mathrm{~nm}$ contribute to this high $\mu_{\mathrm{h}}$ of sample A, as reported in ref. 8 . 

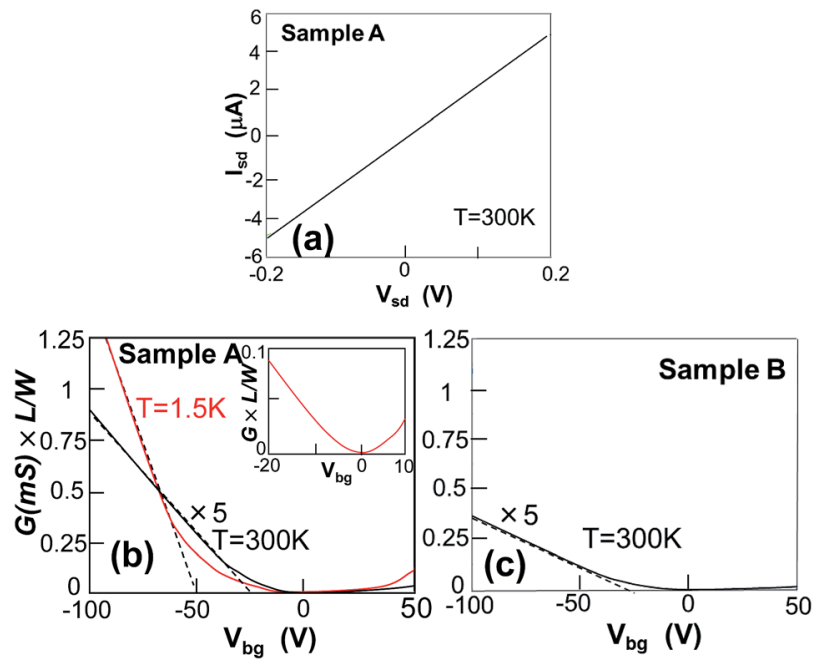

Fig. 3 (a) Drain current $\left(I_{D S}\right)$ as a function of drain voltage $\left(V_{D S}\right)$ of sample A in Fig. 2(d). (b and c) Relationships for conductance (G) vs. back gate voltage $\left(V_{\mathrm{bg}}\right)$ normalized by channel length $(L)$ and width $(W)$ of samples A (b) and B (c). Dotted lines are for extraction of field-effect mobility. Inset of (b): expansion around $V_{\mathrm{bg}}=0$ of the main panel.

These results suggest strong anisotropy in electronic structures, possibly associated with the anisotropic atomic structure of BP, when it is assumed that device orientations are occasionally aligned to the crystal orientations (i.e., directions of the main Hall bars (current channel) in samples A and B in Fig. 2(c) are aligned to the $x$-axis and $y$-axis in Fig. $1(\mathrm{a})$, respectively). Indeed, $t^{\prime}=3 t$ has been reported in BP nanoribbons as mentioned above $\left(e . g\right.$.,$|t|=1.220 \mathrm{eV}$ for in-plane zigzag and $t^{\prime}=$ $3.665 \mathrm{eV}$ for buckling armchair). ${ }^{6}$ This is exactly consistent with the observed anisotropic ratio of $\mu_{\mathrm{h}}$ of $\sim 120 / 320 \mathrm{~cm}^{2} \mathrm{~V}^{-1} \mathrm{~s}^{-1}$ at $T=300 \mathrm{~K}$. The $m^{*}$ value, which is several times smaller in the armchair direction and causes a larger $\mu_{\mathrm{h}}$ to the armchair direction, has actually been reported previously. The ratio of $\mu_{\mathrm{h}}$ of $\sim 120 / 320 \mathrm{~cm}^{2} \mathrm{~V}^{-1} \mathrm{~s}^{-1}$ is smaller than that of the angledependent conductivity ratio of $\sim 0.3 / 0.45 \mathrm{mS} \mathrm{mm}^{-1}$ reported in ref. 8. This also supports a stronger anisotropic electrical property in our sample due to the exact alignment of the Hall bar directions with the crystal directions as mentioned above. Moreover, the band gap closes (i.e., parabolic-like $V_{\mathrm{bg}}$ dependence) around $V_{\mathrm{bg}}=0$ in sample A, as observed in the inset of Fig. 3(b). This should be associated with uniaxial stretch mode as mentioned in later, although it is in contradiction to the presence of the band gap in conventional BP and unlike that of the Dirac point in graphene (i.e., linear $V_{\mathrm{bg}}$ dependence for band gap closing).

Fig. 4(a) exhibits $R_{x y}$ observed in low- $B$ Hall measurements in sample A. As $B$ increases from $B=1.5 \mathrm{~T}, R_{x y}$ monotonically increases but shows three plateaus. The background, in which the mid $B$ points of the individual plateaus are selected and combined (red dotted curve), follows $B^{2 / 3}$ dependence rather than the linear $B$ dependence (blue linear line). This is more evident in the inset, in which the $x$-axis corresponds to $B^{2 / 3}$. In contrast, $\mathrm{SdH}$ oscillations are not evidently observed in $R_{x x}$ in Fig. 4(a) owing to $\mu_{\mathrm{h}}$ being smaller than that of ref. 3 and having
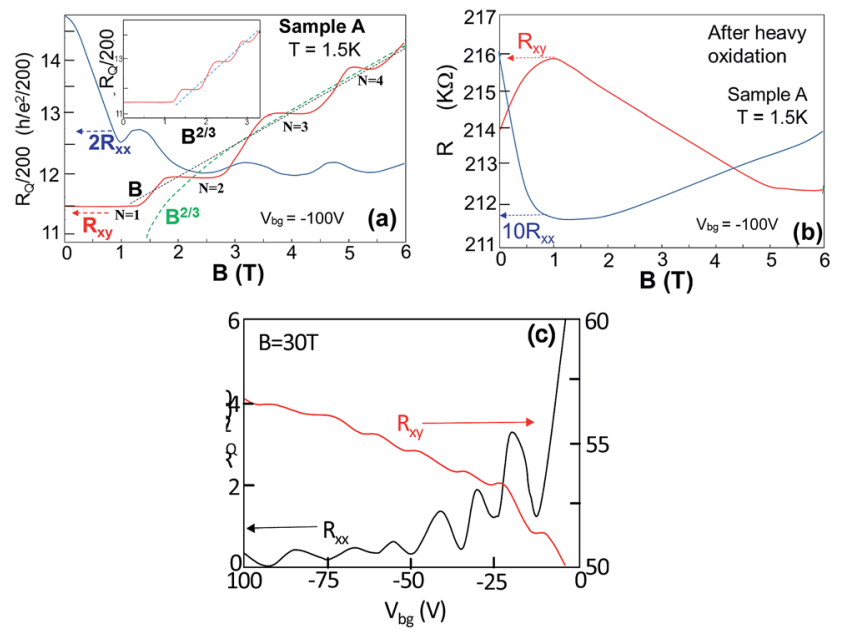

Fig. 4 (a) Observed results in low- $B$ Hall measurements of sample A, $2 R_{x x}$ (blue curve) and $R_{x y}$ (red curve). $N$ noted for $R_{x y}$ indicates the number of the plateaus. Green and black dotted curves correspond to data fit using $B^{2 / 3}$ and $B$ dependence, respectively. (b) Results of Hall measurements of sample $A$ after heavy oxidation by exposing the sample to air atmosphere for one-half of a day, after the Hall measurements in (a). (c) Measurement result of $V_{\mathrm{bg}}$ dependence of $R_{x y}$ and $R_{x x}$ at a fixed high $B(=30 \mathrm{~T})$.

a low- $B$ measurement, although several small oscillations corresponding to the $B$ for the transition regions between the plateaus in $R_{x y}$ are observable. Moreover, the quantized $R_{x y}$ values correspond to $R_{\mathrm{Q}}\left(=h / e^{2}=25.8 \mathrm{k} \Omega\right) / 400-65 \Omega$ between the $N=1$ and $N=2$ plateaus and $R_{\mathrm{Q}} / 200-130 \Omega$ between the $N=$ 2 and $N=3$ plateaus and also the $N=3$ and $N=4$ plateaus. Such small quantization has also been observed in ref. 3 . These resistance behaviours have been entirely smeared out above $T \sim$ $10 \mathrm{~K}$. After heavy oxidation by exposing the sample in air atmosphere for one-half of a day, the $R_{x y}$ changes to an electrondoped type, and the $B^{2 / 3}$ relationship and these plateaus disappear (Fig. 4(b)).

A measurement result of $V_{\mathrm{bg}}$ dependence of $R_{x y}$ and $R_{x x}$ at a fixed high $B(30 \mathrm{~T})$ is shown in Fig. $4(\mathrm{c})$. It demonstrates that our system can possess LLs and $\mathrm{SdH}$ at high $B$, whereas the signature of the hybridization of Schrödinger and Dirac systems cannot be confirmed in such a high $B$ region.

\section{Discussion}

We discuss the $B^{2 / 3}$ dependence of $R_{x y}$ and quantized values $R_{\mathrm{Q}} /$ $N(N=200,400)$ observed in Fig. 4(a). As mentioned in the introduction, $B^{2 / 3}$ dependence of LL energy $E$ has been predicted for the case of $t \neq \mathrm{t}^{\prime}$ under low $B$. It predicts the presence of the $E \propto B^{2 / 3}$ relationship for each $N$ value (node of edge states), i.e., $E \propto[(N+1 / 2) B]^{2 / 3}$. Under a fixed chemical potential $\mu_{\mathrm{c}}$ (i.e., fixed $V_{\mathrm{bg}}$; blue dotted line in Fig. 5), an increase in $R_{x y}$ with increasing $B$ corresponds to a transition between $N$ and $(N$ +1) LL modes in the $E v s$. $[(N+1 / 2) B]^{2 / 3}$ relationship (Fig. 5) and subsequent appearance of individual $R_{x y}$ plateaus. Widths (i.e., period) of individual plateaus in Fig. 4(a) are determined by the $B$ values for this transition between $N$ and $(N+1)$ LL mode, which reflects the $E v s$. $[(N+1 / 2) B]^{2 / 3}$ relationship for individual 


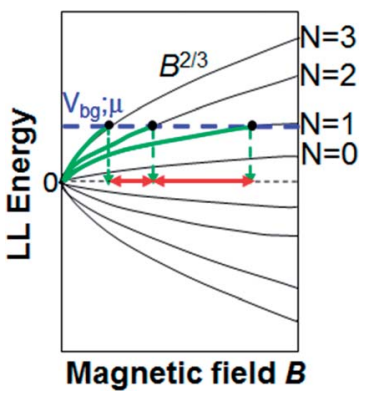

Fig. 5 Schematic of LL energy $E$ vs. $B^{2 / 3}$ relationship at low $B . V_{\text {bg }}$ (blue dotted line) indicates an example of position of the fixed chemical potential $(\mu)$

$N$ values (i.e., green curves along the $B^{2 / 3}$ curves and red arrows along $E=0$ in Fig. 5). Consequently, these $B$ values corresponding to the distance between the mid $B$-points of individual plateaus as mentioned above and quantized values $R_{\mathrm{Q}} / N(N=$ $200,400)$ on the $y$-axis qualitatively display the observed $B^{2 / 3}$ dependence of $R_{x y}$ in Fig. 4(a).

Quantitatively, LL energy $E_{n}=\left(2 \pi e c / \beta(m)^{1 / 2}\right)^{2 / 3}[(N+\gamma) B]^{2 / 3}$ has been theoretically given in ref. 14, where $c$ is the velocity of light and $\beta=4.94$, resulting in the order of $E_{n} \sim 400[(N+1 / 2) B]^{2 / 3}$. Assuming a fixed $E_{n}=\mu$ determined by a fixed $V_{\mathrm{bg}}$, the plateau width should decrease with a ratio of $(0.54 / 2.8)$ for $(N=1 / N=0)$ following this relationship (Fig. 5). On the contrary, the width ratio is almost half in Fig. 4(a), and the quantitative agreement is poor. Moreover, the confirmed anisotropy in $\mu_{\mathrm{h}}$ as large as $\sim 120 / 320 \mathrm{~cm}^{2} \mathrm{~V}^{-1} \mathrm{~s}^{-1}$ at $T=300 \mathrm{~K}$ indicates the possibility for $t^{\prime}$ $=3 t$. For the case of $t^{\prime}>2 t$, ref. 14 predicts that a crossover should appear between a quadratic and a quartic region. Therefore, further experimental and theoretical investigations are required for qualitative conclusion.

Mono (few)-layer BP has conventionally no Dirac cones (Dirac spectrum), whereas the closed band gap and subsequent appearance of the Dirac cone have been recently reported in potassium-doped BP, as mentioned above. ${ }^{9,10}$ It exhibited strong anisotropy (i.e., a Dirac semimetal state with a linear band dispersion in the armchair direction (for the Dirac spectrum) and a quadratic one in the zigzag direction (for the Schrödinger spectrum)). Moreover, under strong strain (stress, bending) and high electrical fields, similar band gap modulations have been reported. ${ }^{11-13,17}$ In the present case, introduction of strain has been confirmed by the Raman spectrum (Fig. 2(c)). The observed shift of $A_{g}^{1}$ and $A_{g}^{2}$ peaks corresponds to those in uniaxial stretch mode with $\sim 0.1 \varepsilon$ along the armchair direction in $\mathrm{BP} .{ }^{24}$ It is well known that cutting the $\mathrm{SiO}_{2} / \mathrm{Si}$ substrate and bonding the wafer of thinned substrates introduce such uniaxial strains (stress). ${ }^{18-21}$ On the other hand, closed band gap in BP is predicted by applying a uniaxial strain along the armchair direction (e.g., $0.1-0.16 \varepsilon){ }^{13}$ The observed uniaxial stretch mode with $\sim 0.1 \varepsilon$ along the armchair direction corresponds to this value, and thus, it implies the possibility of closed band gap along the armchair direction and subsequent emergence of a Dirac spectrum. It is approximately consistent with the closed band gap around $V_{\mathrm{bg}}=0$ shown in the inset of Fig. 3(b).
The observed quantized $R_{x y}$ values $\sim R_{\mathrm{Q}} / 200$ (or 400 ) are extremely small and the origin is not clear at this moment. These are attributed to neither quantum Hall effect (as mentioned above) nor universal conductance fluctuation (UCF), in which the root-mean-square (rms) amplitude of the fluctuation $\delta G$ reaches the universal value $\sim e^{2} / h$, when the sample size $L \ll$ both $L_{\phi}$ (phase coherence length) and $L_{\mathrm{T}}$ (thermal length). When either $L_{\phi}$ or $L_{\mathrm{T}}$ becomes smaller than $L$, the shorter of these governs the amplitude of fluctuations. The former corresponds to our case because of a small BP flake. In contrast, the observed small $R_{x y}$ step is not fluctuation, and $\delta G$ (i.e., conductance peak) cannot be defined. Even if we assume the $R_{x y}$ step $\sim R_{\mathrm{Q}} / 200$ as $1 / \delta G, \delta G$ becomes $\sim 200 \times\left(1 / R_{\mathrm{Q}}\right)$. This value cannot be explained by the universal value $\sim e^{2} / h=1 / R_{\mathrm{Q}}$.

Possibilities of similar $t \neq t^{\prime}$ relationship and subsequent appearance of $B^{2 / 3}$ dependence of LLs in other systems have also been reported in the above-mentioned graphene within uniaxial stress (bending), ${ }^{17} 2 \mathrm{D}$ organic conductors, ${ }^{22}$ and cold atoms in a laser-beam induced optical lattice. ${ }^{23}$ Strong anisotropic atomic structure of BP with $t \neq t^{\prime}$ may be favored to yield $B^{2 / 3}$ dependence of LLs compared with these systems because of the presence of the $t \neq t^{\prime}$ relationship.

\section{Conclusions}

In conclusion, we have reported the observation of $B^{2 / 3}$ dependence of $R_{x y}$ with extremely small quantized levels, which are related to anisotropic electronic structure confirmed by strongly anisotropic $\mu_{\mathrm{h}}$ in few-layer BP. Hybridization of a possible Dirac spectrum (arising from a closed band gap introduced by the uniaxial stretch along the armchair direction, which was confirmed by the Raman spectrum) and Schrödinger spectrum along the zigzag direction is one of the possible qualitative interpretations for the $B^{2 / 3}$ dependence. We have found such unique characteristics only in a few samples to date, in which the current channel direction was occasionally aligned to the exact armchair direction and also the closed band gap was accidentally realized by uniaxial stretch mode through the $\mathrm{Si} /$ $\mathrm{SiO}_{2}$ substrate cutting and wire-bonding processes. Therefore, intentional fabrication of such samples as well as confirmation of the uniaxial Dirac spectrum and the values of $t$ and $t^{\prime}$ in oxidized BP is indispensable. Investigation of the case for mono-layer BP is also indispensable using a hBN substrate. Nevertheless, the observed phenomena are robust and imply significant potential due to strong and unique anisotropy of atom-thin (few-layer) BP, which is highly expected to open doors to novel physics and applications for innovative 2D atom-thin devices.

\section{Acknowledgements}

The authors thank T. Nakamura, S. Katsumoto, Y. Shimazaki, M. Yamamoto, S. Tarucha, T. Enoki, A. H. Macdonald, P. Seneor, R. Wiesendanger, P. Herrero, P. Kim, and M. S. Dresselhaus for their technical contribution, fruitful discussions, and continuous encouragement. The study at Aoyama Gakuin was partly supported by a Grant-in-aid for Scientific 
Research (15K13277) in MEXT and AOARD grant (135049) in U.S. Air Force Office of Scientific Researches.

\section{Notes and references}

1 S. P. Koenig, R. A. Doganov, H. Schmidt, A. H. Castro Neto and B. Özyilmaz, Appl. Phys. Lett., 2014, 104, 103106.

2 A. C. -Gomez, L. Vicarelli, E. Prada, J. O. Island, K. L. N. Acharya, S. I. Blanter, D. J. Groenendijk, M. Buscema, G. A. Steele and J. V. Alvarez, 2D Materials, 2014, 1, 025001.

3 L. Li, G. J. Ye, V. Tran, R. Fei, G. Chen, H. Wang, J. Wang, K. Watanabe, T. Taniguchi, L. Yang, et al., Nat. Nanotechnol., 2015, 10, 608-613.

4 Y. Nakanishi, R. Iwaki, D. Soriano, S. Roche, T. Nakamura, S. Katsumoto and J. Haruyama, Nano Res., 2016, 10, 718-728. 5 Z. Zhu, C. Li, W. Yu, D. Chang, Q. Sun and Y. Jia, Appl. Phys. Lett., 2014, 105, 113105.

6 A. N. Rudenko and M. I. Katsnelson, Phys. Rev. B: Condens. Matter Mater. Phys., 2014, 89, 201408.

7 E. T. Sisakht, M. H. Zare and F. Fazileh, Phys. Rev. B: Condens. Matter Mater. Phys., 2015, 91, 085409.

8 H. Liu, A. T. Neal, Z. Zhu, Z. Luo, X. Xu, D. Tománek and P. D. Ye, ACS Nano, 2014, 8, 4033-4041.

9 J. Kim, S. S. Baik, S. H. Ryu, Y. Sohn, S. Park, B.-G. Park, J. Denlinger, Y. Yi, H. J. Choi and K. S. Kim, Science, 2015, 349, 723.

10 S. S. Baik, K. S. Kim, Y. Yi and H. Joon Choi, Nano Lett., 2015, 15, 7788.

11 A. S. Rodin, A. Carvalho and A. H. Castro Neto, Phys. Rev. Lett., 2014, 112, 176801.

12 X. Peng, Q. Wei and A. Copple, Phys. Rev. B: Condens. Matter Mater. Phys., 2014, 90, 085402.

13 Y. Li, S. Yang and J. J. Li, J. Phys. Chem. C, 2014, 118, 2397023976.

14 P. Dietl, F. Piéchon and G. Montambaux, Phys. Rev. Lett., 2008, 100, 236405.
15 K. S. Novoselov, A. K. Geim, S. V. Morozov, D. Jiang, Y. Zhang, S. V. Dubonos, I. V. Grigorieva and A. A. Firsov, Science, 2004, 306, 666.

16 K. S. Novoselov, A. K. Geim, S. V. Morozov, D. Jiang, Y. Zhang, S. V. Dubonos, I. V. Grigorieva and A. A. Firsov, Nature, 2005, 438, 197.

17 R. Heyd, A. Charlier and E. McRae, Phys. Rev. B: Condens. Matter Mater. Phys., 1997, 55, 6820.

18 K. T. Turner, ECS Trans., 2008, 16, 321-328.

19 W. N. Sharpe Jr, W. N. Sharpe Jr, J. Pulskamp, J. Pulskamp, D. S. Gianola, C. Eberl, C. Eberl, R. G. Polcawich, R. G. Polcawich and R. J. Thompson, Exp. Mech., 2006, 47, 649.

20 S. L. Toh, K. P. Loh, C. B. Boothroyd, K. Li, C. H. Ang and L. Chan, J. Vac. Sci. Technol., B: Microelectron. Nanometer Struct.-Process., Meas., Phenom., 2005, 23.3, 940.

21 S. Goel, A. Kovalchenko, A. Stukowski and G. Cross, Acta Biomater., 2016, 105, 464.

22 S. Katayama, A. Kobayashi and Y. Suzumura, J. Phys. Soc. Jpn., 2006, 75, 054705.

23 E. Zhao and A. Paramekanti, Phys. Rev. Lett., 2006, 97, 230404.

24 R. Fei and L. Yang, Appl. Phys. Lett., 2014, 105, 083120.

25 Y. Katagiri, T. Nakamura, A. Ishii, C. Ohata, M. Hasegawa, S. Katsumoto, T. Cusati, A. Fortunelli, G. Iannaccone, G. Fiori, S. Roche and J. Haruyama, Nano Lett., 2016, 16, 3788.

26 Y. Hasegawa and M. Kohmoto, Phys. Rev. B: Condens. Matter Mater. Phys., 2006, 74, 155415.

27 V. Tran, R. Soklaski, Y. Liang and L. Yan, Phys. Rev. B: Condens. Matter Mater. Phys., 2014, 89, 235319.

28 R. Fei, V. Tran and L. Yang, Phys. Rev. B: Condens. Matter Mater. Phys., 2015, 91, 195319. 